\title{
EPILEPTIFORM EEG DISCHARGES AND RISK OF EPILEPSY FOLLOWING FEBRILE SEIZURE
}

Investigators at Kangnam and Masan Samsung Changwon Hospitals, Korea, studied the relation between epileptiform discharges on the EEG after febrile seizures (FS) and the risk of developing epilepsy and recurrence of FS. Thirty-six children with FS and EEG epileptiform discharges were compared with a control group of 87 children with FS and normal EEG. The average age of the abnormal EEG group was higher than the control group (39.4 vs 25.9 months; $p<0.05$ ). First EEGs were obtained 4.4 and 4.5 days after the FS in study and control groups, respectively. Acute treatment with lorazepam was the same for both groups. FS were simple in $43 \%$ of the abnormal EEG group and 53\% of the control group (NS). Complex FS occurred in 57\% of the abnormal EEG group and $47 \%$ of the controls (NS). Recurrence rate of FS in patients with normal EEG $(26.4 \%)$ was not significantly different from that in patients with FS and EEG showing epileptiform discharges $(33.3 \%)$. Of patients with epileptiform discharges on EEG, $25 \%$ (9 of 36) had epilepsy compared to $2.3 \%$ (2 of 87 ) of children in the control group, and the difference was significant. EEG epileptiform discharges were focal spikes (central in 56\%); none showed generalized spikes. Follow-up EEG in 19 patients (53\%) with repeated febrile and unprovoked seizures was normal in $8(32 \%)$ and showed persistent epileptiform discharges in $11(68 \%)$. Neuroimaging studies in both groups were normal. Epileptiform discharges on the EEG of FS patients are a predictive risk factor of the development of epilepsy. (Wo SB, Lee JH, Lee YJ, Sung, T-J, Lee KH, Kim SK. Risk for developing epilepsy and epileptiform discharges on EEG in patients with febrile seizures. Brain Dev 2013 Apr;35(4):307-11). (Response: Dr Sung Koo Kim, Division of Pediatric Neurology, Kangnam Sacred Heart Hospital, Hallym University, Seoul 150-950, R of Korea. E-mail: pedkimsk@gmail.com).

COMMENT. In the current study, further analysis of the data reveals that the incidence of EEG showing epileptiform discharges in patients presenting with FS is $29 \%$ (36 of 123) and the incidence of epilepsy is 9\% (11 of 123). Follow-up EEGs had persistent epileptiform discharges in $11(68.4 \%)$ patients, and $8(73 \%)$ of the 11 had recurrent unprovoked seizures. In a review of the literature from 1947 through 1964, the mean incidence of EEG epileptiform discharges in 23 published reports of children with FS was 25\% (Millichap JG, et al. Neurology 1960 Jul;10:643-53; Febrile Convulsions. New York, Macmillan, 1968). In one early study (Lennox MA. Proc Assoc Res Nerv Ment Dis 1947;26:342-365) the incidence of epilepsy in patients with FS and paroxysmal EEGs was 53\%. Since the incidence of abnormal EEGs was higher in older children, Lennox recommended repeated EEGs following a febrile seizure to determine the risk of development of epilepsy. Patients with pronounced slow frequencies in the EEG had a greater incidence of recurrent FS and paroxysmal abnormalities. EEG is rarely indicated following a simple FS but may be of predictive value in development of epilepsy following a complex, especially focal FS. 